\title{
MEN 2A - a rare syndrome with variable intrafamilial gene expressivity, case presentation
}

\author{
Authors: \\ Ana Maria Hilma ${ }^{2}$, Adriana Gogoi ${ }^{3}$, Simona Jercalau ${ }^{1}$, Anda Dumitrascu ${ }^{1}$, Andrei Goldstein ${ }^{1}$, Corin Badiu ${ }^{1,4}$ \\ 1. National Institute of Endocrinology "C.I. Parhon", Bucharest, Romania \\ 2. Geviana Medica Company \\ 3. Medicover Private Hospital \\ 4. "Carol Davila" University of Medicine, Bucharest, Romania
}

\section{Introduction:}

- MEN2A is an autosomal dominant inherited syndrome, caused by a gain of function germline mutation in the RET proto-oncogene, with multiglandular tumoral development.

- The majority of mutations involve one of the six cysteine residues in the cysteine-rich region of the RET protein's extracellular domain, encoded in the exons 10 and 11.

- Although the presence of MTC is very high and $50 \%$ of patients present with pheochromocytoma, the penetrance of hyperparathyroidism is estimated to be between 9 to $34 \%$.

- The clinical presentation of the syndrome varies widely even in members of the same family, because of the difference of gene penetration(1).

- In $5 \%$ of cases, hyperparathyroidism may be the first manifestation and it is usually mild.

\section{Case Presentation:}

We present a patient with MEN2A, yet to be confirmed as a family case:

1. MT, 53 yo woman, presented in our clinic in 2017 for medical evaluation. She was known with pheochromocytoma diagnosed at the age of 31, Graves disease with multinodular goiter, and operated breast cancer at 48 yo. She had normal urinary and plasma MN and NMN values, normal calcium, phosphate and PTH levels, and high calcitonin value $(81 \mathrm{pg} / \mathrm{ml})$. In the presence of a high risk thyroid nodule on ultrasound, total thyroidectomy was performed and MTC was confirmed. Recently, genetic testing revealed the presence of RET 11 Cys634Trp mutation and MEN2A was confirmed.

2. Her sister, $\mathrm{BC} 49$ yo, known with multiple melanomas, was subsequently evaluated and primary hyperparathyroidism due to parathyroid adenoma was diagnosed in the presence of repeated high calcium (10.7/10.61/10.8 mg/dl), high normal PTH (62 pg/ml) and normal 25-OH-vitamin D levels. MTC and pheocromocytoma were excluded and genetic testing result is in pending.

\begin{tabular}{|c|c|c|}
\hline & MT, 53 yo woman & BC 49 yo, her sister \\
\hline Other malignancies & breast cancer & multiple melanomas \\
\hline Pheochromocytoma & yes & no \\
\hline MTC & Yes & no \\
\hline HPTH & no & Yes \\
\hline Genetic test & RET 11 Cys634Trp mutation present confirming MEN2A & Pending \\
\hline
\end{tabular}

\section{Discussions:}

What is the correct diagnosis for the second case? Does this patient with a solitary parathyroid adenoma have a rare form of MEN2A syndrome (without parathyroid hyperplasia) or is it just a sporadic disease, in a family with different associated malignancies (breast cancer, malignant melanomas), where the RET mutation was confirmed in her sister? 